\title{
Interactive comment on "On the interaction of short linear waves with internal solitary waves" by Chengzhu Xu and Marek Stastna
}

\author{
K. Terletska (Editor) \\ kterletska@gmail.com \\ Received and published: 12 October 2017
}

Understanding of dynamics of interaction between a fully nonlinear internal solitary waves and linear internal short waves, estimation of energy transfer during two types of interaction (head-on collision and overtaking) are of great importance for understanding of the nonlinear internal wave dynamics. The paper highlighted properties of nonlinear internal solitary waves that are new and interesting. The topic, title, abstract and text of the paper are appropriate for the NPG Journal. I also have some comments:

1. Title: "On the interaction of short linear waves with internal solitary waves" It should be mentioned that short linear waves are also internal.

2. Page.6. Could you please explain in more detail estimation of wavelength. It is not 
clear.

3. Page 6. "The amplitude of linear waves is set to be $1 \mathrm{~mm}(!)$ for all cases." - It seems NPGD it is the mistake, too small amplitude. I guess that the amplitude of linear internal waves was $1 \mathrm{~cm}$. And the vertical resolution is about $1 \mathrm{~mm}$.

4. Than the question arises: Is the resolution fine enough to resolve linear internal short waves?

5. Page 7 " In fact, simulations with an amplitude of $2 \mathrm{~mm}(!)$ have produced quantitatively similar results, and thus will not be discussed in this paper."- Same mistake. Probably "amplitude is $2 \mathrm{~cm}$ "

6. Page 7. What is the value $L x$ in the equation (8)

7. Page 9. Fig. 4. Why ISW in this figure moves backward? In Fig. 4. (c) at $t=146 \mathrm{~s}$ the ISW has shifted backward to $x=7.2$. Because on the previous frame in Fig.4. (b) ISW was located around $x=9.7$ at $t=73 \mathrm{~s}$.

8. Page 16 line 5 mistake: "the linear waves have an amplitude of $0.2 \mathrm{~m}$ (!) " Probably "amplitude is $2 \mathrm{~cm}$ "

9. I didn't find the value of amplitudes of mode-2 waves.

Interactive comment on Nonlin. Processes Geophys. Discuss., https://doi.org/10.5194/npg2017-51, 2017. 